\title{
LA ESTRATEGIA "EL ZORRO Y LAS OVEJAS" EN LA RESOLUCIÓN DE PROBLEMAS ADITIVOS EN NIÑOS Y NIÑAS DEL SEGUNDO GRADO DE PRIMARIA
}

\section{THE STRATEGY "THE FOX AND THE SHEEP" IN THE RESOLUTION OF ADDITIVE PROBLEMS IN CHILDREN OF THE SECOND GRADE OF PRIMARY SCHOOL}

\author{
DOI: Https://doi.org/10.53595/rlo.2021.1.003
}

\author{
Gladys Ochin Quispe Quispe \\ Universidad Nacional del Altiplano \\ gladysayane@gmail.com
}

\author{
Yaqueline Bejar Mamani \\ Universidad Nacional del Altiplano \\ yabema.4asm@gmail.com
}

\section{RESUMEN}

La presente investigación tiene como objetivo general establecer la eficacia de la estrategia "el zorro y las ovejas" en la resolución de problemas aditivos en niños del segundo grado de la IEP N 72308 “Juan Bustamante Dueñas" de Pusi en el año 2019. Metodológicamente es de tipo experimental, nivel cuasi-experimental, diseño de dos grupos con pre-prueba y pos-prueba; la muestra estuvo constituida por 23 estudiantes, a quienes se les aplicó las pruebas respectivamente. Los resultados obtenidos en los procesos de resolución de problemas aditivos muestran que la mayoría de los estudiantes que pertenecen al grupo experimental del segundo grado en la prueba de entrada obtuvieron un promedio de 0,9 puntos estando en inicio del aprendizaje, mientras que en la prueba de salida, luego de aplicar la estrategia "el zorro y las ovejas" mediante las sesiones de aprendizaje por un periodo de un mes, alcanzaron un promedio de 16,41 puntos, ubicándolos en los niveles de logro (A) y logro destacado (AD). Para la prueba de la hipótesis se utilizó el estadístico T de Student en la cual se pude apreciar el valor de $\mathrm{P}=0,025<0,05$, es decir, existe una diferencia significativa entre los promedios antes $\mathrm{y}$ después de la aplicación de la estrategia planteada, con lo cual se evidencia que la hipótesis ha sido confirmada, dichos resultados permitieron establecer la siguiente conclusión: La estrategia "el zorro y las ovejas", resulta eficaz para la resolución de problemas aditivos en niños del segundo grado.

Palabras clave: Estrategia, eficacia, zorro y las ovejas, resolución de problemas, adición.

\section{ABSTRACT}

The present investigation have as objective general establish the effectiveness of the strategy "the fox and the sheep" in problem solving additives in children of second grade of IEP N ${ }^{\circ} 72308$ "Juan Bustamante Dueñas" of Pusi in 2019. Methodologically it is experimental, quasi-experimental level, two group design with pre-test and post-test; the sample was made up of 23 students, to whom it was applied the tests respectively. The 
results obtained in the processes of Problem resolution additives show that most of the students who belong to the experimental group of the second grade in the entrance test got an average 0.9 points being at the beginning of learning, while that in the start test, after applying the strategy "the fox and the sheep" through the learning sessions for a period of one month, they reached an average of 16.41 points, placing them in the achievement levels (A) and achievement featured (AD). For the test of the hypothesis the student's $t$ statistic in which you could appreciate the value of $\mathrm{P}=0.025<0.05$, that is to say, there is a significant difference between the averages before and after the application of the strategy raised, which shows that the hypothesis has been confirmed, sayings results allowed to establish the following conclusion: The strategy "the fox and the sheep", is effective for additive troubleshooting in children of the second grade of the IEP $\mathrm{N}^{\circ} 72308$ "Juan Bustamante Dueñas".

Keywords: Strategy, effectiveness, fox and sheep, problem solving, addition.

\section{INTRODUCCIÓN}

En la actualidad los estudiantes de nuestro país, principalmente en las instituciones educativas regulares de carácter estatal, presentan dificultades en el aprendizaje de las matemáticas, dentro de ello en la resolución de problemas aditivos y la poca importancia que se le da al empleo de recursos didácticos y estrategias en las sesiones de clase; asimismo sabemos por propia experiencia, de ahí surge la necesidad de perfeccionar estrategias y métodos para la enseñanza de la matemática, y que por muchos años primaron las tendencias abstractas, hoy en día surgieron cambios hacia lo intuitivo, lo concreto operativo y el trabajo en equipo. La enseñanza de la matemática de nuestro país, en la mayoría de los casos se ha convertido en una asignatura de tortura mental, tan alejada de la realidad y del contexto social, mostrando una pérdida de la capacidad de análisis y creatividad en los estudiantes al solucionar problemas de su contexto social.

En el Perú, uno de los grandes problemas que afrontamos los docentes es el bajo rendimiento que tienen los estudiantes en el área de Matemática, los resultados se pueden observar en las diferentes pruebas del programa Internacional de Evaluación de Estudiantes (PISA), auspiciado por la (UNESCO) y la Organización para la Corporación y el Desarrollo Económico (OCDE); donde participan diferentes países del mundo y de América Latina, evaluando los dominios en diferentes áreas (Matemática, comprensión lectora y competencias científicas), Los resultados fueron deficientes para el Perú, ocupando últimos lugares. Así en PISA (2018), de 77 países participantes, ocupó el lugar 64, obteniendo un promedio de 400 puntos.

En el Informe Nacional, los resultados de matemáticas no son tan satisfactorios quedando de la siguiente manera en el examen (ECE) Evaluación Censal de Estudiantes. (2018): 
Urbano: El 52,5\% en inicio, $32,1 \%$ en proceso y el 15,3\% en satisfactorio. Rural: El $74,3 \%$ en inicio, $16,4 \%$ en proceso y el $9,3 \%$ en satisfactorio. (pág. 15)

El informe de la DRE-Puno, los resultados en matemáticas no son tan satisfactorios quedando de la siguiente manera el último examen (ECE) Evaluación Censal de Estudiantes - 2018. (DRE, 2018): Urbano: El 5,4\% en proceso de inicio, 14,8\% en inicio, $41,2 \%$ en proceso y el 38,6\% en satisfactorio. Rural: El 5,2\% en proceso de inicio, $15,1 \%$ en inicio, $42,9 \%$ en proceso y el $36,8 \%$ en satisfactorio.

La IEP N 72308 “Juan Bustamante Dueñas” de Pusi no se hace ajena a esta problemática, ya que se ha observado en los estudiantes muchas dificultades en su aprendizaje, especialmente en el área de matemática lo cual es un hecho alarmante pues evidencia que los estudiantes no presentan un interés o necesidad por aprender matemática, porque desconocen lo útil que es para su vida, mostrando un bajo nivel de desempeño en la resolución de problemas aditivos, esto debido a que los docentes mantienen el esquema de enseñanza tradicional y además hay incertidumbre sobre la aplicación de estrategias y el manejo de los materiales didácticos ya que no se aprovecha al máximo los materiales que les da el Estado.

Para Godino , Batanero, \& Font, (2003): "conocer" o "saber" matemáticas, es algo más que repetir las definiciones o ser capaz de identificar propiedades de números... La persona que sabe matemáticas ha de ser capaz de usar el lenguaje y conceptos matemáticos para resolver problemas. No es posible dar sentido pleno a los objetos matemáticos si no los relacionamos con los problemas de los que han surgido”. (pág. 66) La actividad de resolver problemas es esencial si queremos conseguir un aprendizaje significativo de las matemáticas. No debemos pensar en esta actividad sólo como un contenido más del currículo matemático, sino como uno de los vehículos principales del aprendizaje de las matemáticas, y una fuente de motivación para los alumnos ya que permite contextualizar y personalizar los conocimientos. Al resolver un problema, el alumno dota de significado a las prácticas matemáticas realizadas, ya que comprende su finalidad. (Godino et al, 2003).

La importancia que se da a la resolución de problemas en los currículos actuales, es el resultado de un punto de vista sobre las matemáticas que considera que su esencia es precisamente la resolución de problemas. Muchos autores han ayudado a desarrollar este punto de vista como, por ejemplo, destaca Polya. Para este autor, la resolución de un problema consiste, a grandes rasgos, en cuatro fases: 1) Comprender el problema, 2) Concebir un plan, 3) Ejecutar el plan y 4) Examinar la solución obtenida. Cada fase se 
acompaña de una serie de preguntas cuya intención clara es actuar como guía para la acción (Godino et al, 2003)

El término resolución de problemas ha servido como un paraguas bajo el cual se realizan radicalmente diferentes tipos de investigación. Un problema de matemáticas es una situación real o ficticia que puede tener interés por sí misma, al margen del contexto, que involucra cierto grado de incertidumbre, implícito en lo que se conoce como las preguntas del problema o la información desconocida, cuya clarificación requiere la actividad mental y se manifiesta en un sujeto, al que llaman resolutor.

Por otro lado la etnomatemática se define como un conjunto de conocimientos de un grupo sociocultural identificable, en el marco de su cosmovisión, que se manifiesta a través de las siguientes actividades: medir, contar, localizar, diseñar, jugar y explicar.

Los conocimientos etnomatemáticos se expresan mediante símbolos, que incluyen el uso de expresiones en la propia lengua originaria; sin embargo, queremos llamar la atención sobre el hecho que la etnomatemática de una cultura originaria generalmente no se expresa de modo abstracto, sino que está vinculada a determinadas actividades de un grupo sociocultural o comunidad, las mismas que se desarrollan en el marco de su propia visión del mundo. Es importante tener en cuenta que la etnomatemática de cada pueblo tiene un origen distinto. Esto se puede ilustrar con los ejemplos siguientes, dos extraídos del mundo andino, y el otro, del ámbito amazónico. (MINEDU, Matemáticas en educación intercultural bilingüe, 2015, pág. 12)

La Taptana o ajedrez andino, en nuestro contexto conocido como el "zorro y las ovejas", es un juego que se practica desde la época de los incas, se trata del enfrentamiento de un grupo de ovejas contra el zorro. La enseñanza que nos da el juego es "La unión hace la fuerza”, porque la única forma de vencer al zorro es manteniéndose unidos. (Holm, 1945). El zorro y las ovejas es un juego originario de las comunidades puneñas quechuas de Sillota, Chaupi, Sahuacasi y Matazo. Se juega a través de la taptana lúdica o juego de ajedrez incaico. Este juego también se practica en el Cusco.

En las crónicas de Huamán Poma de Ayala se reproduce con un dibujo que él nombra como Taptana o axedrez incaico. La palabra taptana viene de la palabra quechua «tapta» que significa saltar, por esto se asocia este juego al salto que da el zorro para capturar las ovejas. (MINEDU, 2008).

Se puede utilizar para que el estudiante desarrolle su capacidad de razonamiento pues tiene que pensar en las consecuencias de cada una de sus jugadas e imaginar las posibilidades jugadas de su contrincante cada vez que efectúa un movimiento 
determinado de alguna de las piezas las que le corresponde jugar. En este sentido, los jugadores usan constantemente las proposiciones implicativas del tipo: "si...entonces"; es decir, utilizan cadenas de razonamiento.

El juego plantea la búsqueda de estrategias. Un zorro debe atrapar 10 ovejas, y estas diez ovejas quieren cerrar el paso al zorro para que se inmovilice y no las pueda atrapar. El juego se efectúa con una ficha pequeña que representa al zorro y diez fichas más pequeñas que la anterior, que representan las diez ovejas. Uno de los jugadores hace del zorro y el otro conduce las ovejas. Por sorteo, inicia el juego uno de los dos jugadores y luego continuaran por turno.

El zorro se puede desplazar en cualquier dirección, hacia adelante o hacia atrás, siempre un paso, sobre cualquiera de los segmentos de la figura y ubicarse sobre el punto de intersección de dos o más segmentos según corresponda. En cambio, cada oveja solo puede avanzar un paso hacia adelante desplazándose sobre una de las diagonales o sobre uno de los lados de un cuadro las ovejas no pueden retroceder.

El zorro se come a una oveja cuando puede pasar sobre ella saltando sobre el punto de intersección en el que se encuentra la oveja. En este caso se saca del tablero a la oveja correspondiente. El jugador encargado de desplazar al zorro gana el juego si logra comer las diez ovejas.

El jugador que se encarga de las ovejas debe desplazarlas buscando siempre cercar al zorro, de modo que este no pueda dar un paso más sobre ninguno de los segmentos del tablero. Si las ovejas logran cercar al zorro, entonces el jugador encargado de movilizarlas abra ganado el juego.

En cuanto al diagrama del juego el zorro y las ovejas es graficar un tablero en la mesa, en el suelo o extender un tablero en la mesa, se juega entre dos personas, en el juego se puede representar al zorro y las ovejas con piedritas, chapitas, con productos del lugar y otros. El zorro tiene que avanzar en cada jugada una recta en el rectángulo y la oveja de manera del otro lado.

Cuando el jugador que juega con las ovejas se descuida en avanzar en forma conjunta sus ovejas, el zorro da un salto y come una oveja, de esa manera va comiendo las ovejas hasta terminar comer las doce ovejas en donde gana el zorro y cuando el jugador de las ovejas avanza en forma conjunta no se hace comer ninguna oveja al zorro lo arrincona hasta su casa sin dar lugar a moverse en este caso gana el jugador de las ovejas. (MINEDU, 2015). De lo descrito anteriormente es necesario realizar investigaciones concernientes a disminuir la brecha de deficiencia. De tal manera, esta investigación con la estrategia 
planteada, desarrolla competencias satisfactorias relacionadas a la resolución de problemas aditivos en los niños del segundo grado de la IEP N 72308 "Juan Bustamante Dueñas" de Pusi.

\section{MATERIALES Y MÉTODOS}

La investigación se desarrolló en los 3835 m s. n. m. en el Altiplano peruano, Departamento de Puno, provincia de Huancané, distrito de Pusi al Sur de la Provincia, al Norte con los distritos de Taraco y de Samán, este último en la Provincia de Azángaro; al Sur con los de Capachica y Coata; al Este con el Lago; y al Oeste con la provincia de San Román, distritos de Caracoto y de Juliaca en la Institución Educativa Primaria N ${ }^{\circ} 72308$ “Juan Bustamante Dueñas” de Pusi.

La investigación duró dos años desde su planteamiento como proyecto (finales del 2018), su ejecución (del 07 de octubre del 2019 al 29 de octubre del 2019), llegando a culminarse en el informe a finales del 2020

La población de estudio de la presente investigación está conformada por todos los alumnos del segundo grado "A" y "B" de la Institución Educativa Primaria N 72308 "Juan Bustamante Dueñas" que supera el 10\% del total de estudiantes, siendo un total de 23 estudiantes. La presente investigación corresponde al enfoque cuantitativo, de tipo experimental, nivel cuasi-experimental.

\section{RESULTADOS Y DISCUSIÓN}

Primero se presentan los resultados generales obtenidos de la aplicación del juego "el zorro y las ovejas" como estrategia para la resolución de problemas aditivos antes y después del experimento (pre-test y pos-test) en forma de tablas y gráficos para el grupo de control, seguido para el grupo experimental. Luego se comprueba la hipótesis planteada con la aplicación de la prueba T de Student al grupo experimental. Por último, se muestran resultados para el grupo experimental según los procesos de resolución de problemas aditivos.

\section{Resultados de la prueba de entrada (pre test) del grupo experimental y control de los niños de la IEP “Juan Bustamante Dueñas” Pusi-2019}

Como muestra la tabla 1 , el $0.0 \%$ de los estudiantes del grupo experimental y el $9.10 \%$ de los estudiantes del grupo control se encuentran en la categoría (AD) logro destacado del aprendizaje, de la misma manera observamos que el $0.0 \%$ de estudiantes que se encuentran en el grupo experimental y el $36.36 \%$ de los estudiantes del grupo control se encuentran en la categoría (A) logro del aprendizaje, así también se observa en la figura que un $0.0 \%$ de estudiantes del grupo experimental y el $27.27 \%$ de estudiantes que se 
encuentran en el grupo control se encuentran en la categoría (B) en proceso del aprendizaje y por último observamos que un $100 \%$ de estudiantes del grupo experimental y el $27.27 \%$ de los estudiantes del grupo control están en la categoría (C) en inicio del aprendizaje.

Tabla 1: Proceso de Resolución de problemas aditivos de los niños de la IEP “Juan Bustamante Dueñas" Pusi-2019 antes del experimento (pre-test).

\begin{tabular}{|c|c|c|c|c|}
\hline \multirow[b]{2}{*}{ Categoría/escala } & \multicolumn{2}{|c|}{ Grupo experimental } & \multicolumn{2}{|c|}{ Grupo control } \\
\hline & $\begin{array}{l}N^{\circ} \text { de alumnos } \\
\text { (fi) }\end{array}$ & $\begin{array}{l}\text { Porcentaje } \\
(\%)\end{array}$ & $\begin{array}{l}\mathbf{N}^{\circ} \text { de alumnos } \\
\text { (fi) }\end{array}$ & $\begin{array}{l}\text { Porcentaje } \\
(\%)\end{array}$ \\
\hline $\begin{array}{ll}(\mathrm{AD}) & \text { logro } \\
\text { destacado.(18-20) }\end{array}$ & 00 & $0.0 \%$ & 01 & $9.10 \%$ \\
\hline $\begin{array}{l}\text { (A) logro del } \\
\text { aprendizaje.(14-17) }\end{array}$ & 00 & $0.0 \%$ & 04 & $36.36 \%$ \\
\hline $\begin{array}{l}\text { (B) proceso del } \\
\text { aprendizaje.(13-11) }\end{array}$ & 00 & $0.0 \%$ & 03 & $27.27 \%$ \\
\hline $\begin{array}{l}\text { (C) inicio del } \\
\text { aprendizaje.(00-10) }\end{array}$ & 12 & $100 \%$ & 03 & $27.27 \%$ \\
\hline Total & 12 & $100 \%$ & 11 & $100 \%$ \\
\hline
\end{tabular}

En la interpretación realizada, se muestran evidencias objetivas sobre proceso de resolución de problemas aditivos. Si tomamos estos resultados con relación a los objetivos planteados en la investigación, rechazaría la hipótesis planteada. Ahora bien, claro está, la falta de aplicación de estrategias se refleja, los estudiantes si siguen a ese ritmo de aprendizaje en el tema tratado, no tendrán oportunidades para competir con otras instituciones preparadas cuando salgan de la educación básica en los exámenes de clasificación e ingreso a las universidades. Si nos ponemos a analizar estos resultados, comprueban el pésimo rendimiento en las pruebas ECE Y PISA. Si bien es cierto, de las diversas causas existentes, una de ellas es la falta de estrategias de resolución de problemas aditivos en los estudiantes y como explica la UNICEF (2018), que "El juego constituye una de las formas más importantes en las que los niños pequeños obtienen conocimientos y competencias esenciales. Por esta razón, las oportunidades de juego y los entornos que favorecen, la exploración y el aprendizaje práctico constituyen el fundamento de los programas de educación preescolar eficaces".

Proceso en el aprendizaje, durante la ejecución de la estrategia el zorro y las ovejas en los niños y niñas el grupo experimental 
Tabla 2: Resultados de la evolución de aprendizaje en sesiones de aprendizaje durante el proceso experimental.

\begin{tabular}{|c|c|c|c|c|c|c|c|c|}
\hline \multirow[b]{2}{*}{$\begin{array}{l}\text { Sesiones } \\
\text { Categoría }\end{array}$} & \multicolumn{2}{|c|}{ Sesión n ${ }^{\circ} 1$} & \multicolumn{2}{|c|}{ Sesión $n^{\circ} 4$} & \multicolumn{2}{|c|}{ Sesión n ${ }^{\circ} 6$} & \multicolumn{2}{|c|}{ Sesión n ${ }^{\circ} 8$} \\
\hline & (fi) & $(\%)$ & (fi) & $(\%)$ & (fi) & $(\%)$ & (fi) & $(\%)$ \\
\hline $\begin{array}{lr}(\mathrm{AD}) & \text { logro } \\
\text { destacado. }(18-20)\end{array}$ & 0 & $00 \%$ & 1 & $8.33 \%$ & 2 & $16.67 \%$ & 3 & $\begin{array}{c}25.00 \\
\%\end{array}$ \\
\hline $\begin{array}{l}\text { (A) logro del } \\
\text { aprendizaje.(14- } \\
17)\end{array}$ & 4 & $33.33 \%$ & 5 & $41.67 \%$ & 6 & $50.00 \%$ & 6 & $\begin{array}{c}50.00 \\
\%\end{array}$ \\
\hline $\begin{array}{l}\text { (B) proceso del } \\
\text { aprendizaje.(13- } \\
11)\end{array}$ & 5 & $41.67 \%$ & 5 & $41.67 \%$ & 3 & $25.00 \%$ & 3 & $\begin{array}{c}25.00 \\
\%\end{array}$ \\
\hline $\begin{array}{l}\text { (C) inicio del } \\
\text { aprendizaje.(00- } \\
10)\end{array}$ & 3 & $25.00 \%$ & 1 & $8.33 \%$ & 1 & $8.33 \%$ & 0 & $00 \%$ \\
\hline Total & 2 & $100 \%$ & 2 & $100 \%$ & 2 & $100 \%$ & 2 & $100 \%$ \\
\hline
\end{tabular}

En la tabla 2, se representa los resultados de la evolución que tuvieron los estudiantes durante el desarrollo de aprendizaje.

En la sesión 01, se observa que 0 estudiantes que representa el $0.0 \%$ de los estudiantes se encuentran en la categoría (AD), 04 estudiantes que representa el $33.33 \%$ se encuentra en la categoría (A) logro del aprendizaje; 05 de los estudiantes que representa el $41.67 \%$ se encuentra en la categoría (B) en proceso del aprendizaje; y por ultimo 03 estudiantes que representa el $25.00 \%$ se encuentra en la categoría (C) en inicio del aprendizaje.

En la sesión 04, se puede observar que 01 estudiante que representa el $8.33 \%$ de los estudiantes se encuentran en la categoría (AD), 05 estudiantes que representa el $41.67 \%$ se encuentra en la categoría (A) logro del aprendizaje; 05 de los estudiantes que representa el $41.67 \%$ se encuentra en la categoría (B) en proceso del aprendizaje; y por ultimo 01 estudiante que representa el $8.33 \%$ se encuentra en la categoría (C) en inicio del aprendizaje.

En la sesión 06, se puede observar que 02 estudiante que representa el $16.67 \%$ de los estudiantes se encuentran en la categoría (AD), 06 estudiantes que representa el 50.00\% se encuentra en la categoría (A) logro del aprendizaje; 03 de los estudiantes que representa el $25.00 \%$ se encuentra en la categoría (B) en proceso del aprendizaje; y por ultimo 01 estudiante que representa el $8.33 \%$ se encuentra en la categoría (C) en inicio del aprendizaje. 
En la sesión 08, se puede observar que 03 estudiante que representa el $25.00 \%$ de los estudiantes se encuentran en la categoría (AD), 06 estudiantes que representa el $50.00 \%$ se encuentra en la categoría (A) logro del aprendizaje; 03 de los estudiantes que representa el $25.00 \%$ se encuentra en la categoría (B) en proceso del aprendizaje; y por ultimo 00 estudiantes que representa el $00.00 \%$ se encuentra en la categoría (C) en inicio del aprendizaje.

En la interpretación realizada podemos comprobar una mejoría de las puntuaciones obtenidas en el transcurso de las sesiones; al inicio la mayoría tienen calificaciones menores e igual a 10 dentro de la categoría (C) inicio del aprendizaje. En la última sesión realizada se puede apreciar que el $75 \%$ se encuentran en las categorías (A) logro del aprendizaje y (AD) logro destacado del aprendizaje.

Viendo los resultados obtenidos durante las sesiones con la estrategia plateada, observamos que los estudiantes presentan mejorías para la comprensión de problemas, por lo tanto coincidimos con el Ministerio de Educación (2015), dándonos a conocer que la estrategia el "zorro y las ovejas" se puede utilizar para que el estudiante desarrolle su capacidad de razonamiento pues tiene que pensar en las consecuencias de cada una de sus jugadas e imaginar las posibilidades jugadas de su contrincante cada vez que efectúa un movimiento determinado de alguna de las piezas las que le corresponde jugar. En este sentido, los jugadores usan constantemente las proposiciones implicativas del tipo: "si...entonces"; es decir, utilizan cadenas de razonamiento.

\section{Resultados de la prueba de salida (pos-test) del grupo experimental y control de los niños de la IEP “Juan Bustamante Dueñas” Pusi-2019}

Después de haber realizado el experimento, se aplica la prueba escrita de salida (POSTTEST) con el fin de conocer el rendimiento de los niños y niñas. Esta prueba se refiere al contenido correspondiente al área de matemática a falta del aprendizaje o desarrollar la capacidad para resolver problemas aditivos. A continuación, se muestra la siguiente tabla con los datos obtenidos en la prueba de salida.

De la figura mostrada se puede observar que un $91.67 \%$ de estudiantes del grupo experimental se encuentran en la categoría (A) logrando así la eficacia de la estrategia el zorro y las ovejas en la resolución de problemas aditivos.

Coincidiendo así con la conclusión de (Morales, 2011) que menciona que dicha estrategia aplicada, es un valioso aporte al proceso de enseñanza y aprendizaje más adecuado y objetivo y además que Se ha recuperado los juegos andinos, específicamente del "zorro y la oveja" para el aprendizaje de la adición y sustracción. 
Tabla 3: Proceso de Resolución de problemas aditivos de los niños de la IEP "Juan Bustamante Dueñas” Pusi-2019 después del experimento (pos-test).

\begin{tabular}{lcccc}
\hline & \multicolumn{2}{c}{ Grupo experimental } & \multicolumn{2}{c}{ Grupo control } \\
\cline { 2 - 5 } Categoría/escala & $\begin{array}{c}\mathbf{N}^{\circ} \text { de alumnos } \\
(\mathbf{f i})\end{array}$ & $\begin{array}{c}\text { Porcentaje } \\
(\%)\end{array}$ & $\begin{array}{c}\mathbf{N}^{\circ} \text { de alumnos } \\
(\mathbf{f i})\end{array}$ & $\begin{array}{c}\text { Porcentaje } \\
(\%)\end{array}$ \\
$\begin{array}{l}\text { (AD) logro } \\
\text { destacado.(18-20) }\end{array}$ & 01 & $08.33 \%$ & 02 & $18.19 \%$ \\
$\begin{array}{l}\text { (A) logro del } \\
\text { aprendizaje.(14-17) }\end{array}$ & 11 & $91.67 \%$ & 03 & $27.27 \%$ \\
$\begin{array}{l}\text { (B) proceso del } \\
\text { aprendizaje.(13-11) }\end{array}$ & 00 & $0.0 \%$ & 03 & $27.27 \%$ \\
$\begin{array}{l}\text { (C) inicio del } \\
\text { aprendizaje.(00-10) }\end{array}$ & 00 & $0.0 \%$ & 03 & $27.27 \%$ \\
Total & 12 & $100 \%$ & 11 & $100 \%$ \\
\hline
\end{tabular}

Y además Llalla \& Yanque (2015), también afirman que el juego andino, permite en los estudiantes adquirir aprendizajes significativos que les facilita una formación integral además que los juegos andinos no solo cumplen una función de entretenimiento y recreación sino también cumple una importante función socializadora y educativa.

Resultados de los calificativos obtenidos en la prueba de salida de los grupo control y experimental de los niños de la IEP N72308 “Juan Bustamante Dueñas” de Pusi $-2019$

Con estos promedios que se obtiene se deduce que con el material el zorro y las ovejas como estrategia en el aprendizaje de la resolución de problemas en el área de matemática; es decir que este material influye positivamente en el aprendizaje. Esta tabla muestra los calificativos específicos que los estudiantes obtienen con la prueba de salida (PRE TEST) después de haberse realizado el experimento con el grupo control

Tabla 4: Calificativos obtenidos por los estudiantes del grupo control en la prueba de salida.

\begin{tabular}{ccccc}
\hline $\mathbf{X i}$ & $\mathbf{F i}$ & $\mathbf{X i} \mathbf{F i}$ & $\mathbf{X i}^{\mathbf{2}}$ & $\mathbf{X i} \mathbf{i}^{\mathbf{F i}}$ \\
\hline 9 & 2 & 18 & 81 & 162 \\
10 & 1 & 10 & 100 & 100 \\
12 & 2 & 24 & 144 & 288 \\
11 & 1 & 11 & 121 & 121 \\
14 & 2 & 28 & 196 & 392 \\
15 & 1 & 15 & 225 & 225 \\
18 & 2 & 36 & 324 & 648 \\
Total & 11 & 142 & & 1,936 \\
\hline
\end{tabular}


Mediante esta tabla se obtiene la media aritmética:

$$
\bar{x}=\frac{\sum_{i=1}^{n} \text { Xifi }}{\mathrm{n}}=\frac{142}{11}=12.90
$$

Según el resultado de los 11 estudiantes tiene un promedio en media aritmética de 12.9 redondeado al valor inmediato superior a 13 puntos. Lo que significa que los estudiantes del segundo grado sección "A" tiene un promedio de 13 puntos correspondientes y esto fue sin la utilización de la estrategia el zorro y las ovejas.

- Varianza: $\left(\mathbf{s}^{2}\right)$

$$
s^{2}=\frac{\sum_{i=1}^{n} f i\left(x_{i}-\bar{x}\right)}{\mathrm{n}-1}=\frac{1936-(142)^{2} / 11}{11-1}=\frac{1936-1833.09}{10}=10.921
$$

La varianza es de 10.921 redondeado al promedio mayor seria 11 puntos. Este nivel de varianza indica un mínimo y un máximo de promedio.

- Desviación estándar (S)

$$
s^{2}=\frac{\sum_{i=1}^{n} f i\left(x_{i}-\bar{x}\right)^{2}}{\mathrm{n}-1}=10.291=3.20
$$

Se ha obtenido la desviación estándar a través de la raíz cuadrada obteniendo un resultado de 3.20

- Coeficiente de varianza (CV)

$$
\mathrm{CV}=\frac{\mathrm{s}}{\mathrm{x}} 100 \%=\frac{3.20}{12.90} 100=24.80
$$

Podemos afirmar que los datos son regularmente variables

Tabla 5: Calificativos obtenidos por los niños y niñas del grupo experimental en la prueba de salida.

\begin{tabular}{ccccc}
\hline $\mathbf{X i}$ & $\mathbf{F i}$ & $\mathbf{X i} \mathbf{F i}$ & $\mathbf{X i}^{\mathbf{2}}$ & $\mathbf{X i}{ }^{\mathbf{2}} \mathbf{F i}$ \\
\hline 15 & 2 & 30 & 225 & 450 \\
16 & 4 & 64 & 256 & 1024 \\
17 & 5 & 85 & 289 & 1445 \\
18 & 1 & 18 & 324 & 324 \\
\hline Total & 12 & 197 & & 3243 \\
\hline
\end{tabular}


Esta tabla 5 muestra los calificativos específicos que los estudiantes de grupo experimental obtienen con la prueba de salida (POST TEST) después de haberse realizado el experimento.

Mediante esta tabla se obtiene la media aritmética

$$
\bar{x}=\frac{\sum_{i=1}^{n} \text { Xifi }}{\mathrm{n}}=\frac{197}{12}=16.41
$$

Según este resultado los 12 estudiantes obtienen un promedio en media aritmética de 16.41 redondeando al valor inmediato es de 16 lo que significa que los estudiantes del segundo grado sección "B” tiene un promedio de 16.

- Varianza: $\left(s^{2}\right)$

$$
s^{2}=\frac{\sum_{i=1}^{n} f i\left(x_{i}-\bar{x}\right)}{n-1}=\frac{3243-(197)^{2} / 12}{12-1}=\frac{3243-3234.08}{11}=0.810
$$

La varianza es de 0.810 redondeado al promedio mayor seria 1 punto. Este nivel de varianza indica que la varianza del calificativo mínimo y máximo es de aproximado 1 punto con respecto a la media aritmética.

- Desviación estándar (S)

$$
s^{2}=\frac{\sum_{i=1}^{n} f i\left(x_{i}-\bar{x}\right)^{2}}{\mathrm{n}-1}=0.810=0.900
$$

Se ha obtenido la desviación estándar a través de la raíz cuadrada obteniendo un resultado de 0.900 .

\section{- Coeficiente de varianza (CV)}

$$
\mathrm{CV}=\frac{\mathrm{s}}{\mathrm{x}} 100 \%=\frac{0.900}{16.41} 100=5.48
$$

Datos de las medias de tendencia central del grupo experimental y control de los niños de la IEP “Juan Bustamante Dueñas” Pusi-2019

Tabla 6: Medias de tendencia central de los niños del grupo experimental y control.

\begin{tabular}{lll}
\hline Medias de tendencia central & Grupo experimental & Grupo control \\
\hline Promedio o media $(\mathrm{x})$ & 16.41 & 12.90 \\
Varianza $\left(S^{2}\right)$ & 0.810 & 10.92 \\
Desviación estándar (s) & 0.900 & 3.20 \\
Coeficiente de variable (cv) & 5.48 & 24.80 \\
Total & 16.41 & 12.90 \\
\hline
\end{tabular}

En esta tabla 6 se observa que el grupo experimental obtiene un promedio de $16.41 \mathrm{y}$ el grupo control obtiene 12.90. La varianza del grupo experimental es de 0.810 y del grupo 
control es de 10.92. La desviación estándar del grupo experimental es de 0.900 y del grupo control es de 3.20. El coeficiente de variación es de 5.48 para el grupo experimental y para el grupo control es de 24.80 .

\section{Prueba de hipótesis de la investigación}

Se desarrolla mediante la T de Student (comparación de medias) porque:

$\mathrm{N}$ (muestra) < 30 y $\mathrm{N} \in$ a una distribución normal.

Se tiene las siguientes variables:

VI: La estrategia el "zorro y las ovejas".

VD: Resolución de problemas aditivos.

Dos grupos: 1 = control

$$
\mathbf{2}=\text { experimental }
$$

\section{Planteamiento de hipótesis:}

Ho = La estrategia "el zorro y las ovejas", no resulta eficaz para la resolución de problemas aditivos en niños del segundo grado de la IEP $\mathrm{N}^{\circ} 72308$ "Juan Bustamante Dueñas” - Pusi en el 2019.

$\mathbf{H a}=$ La estrategia "el zorro y las ovejas", resulta eficaz para la resolución de problemas aditivos en niños del segundo grado de la IEP $\mathrm{N}^{\circ} 72308$ “Juan Bustamante Dueñas" - Pusi en el 2019.

Índice de confiabilidad al 95\% $\alpha=0,05=5 \%$ (nivel confiable)

$\mathbf{p}=$ Resultado de la significancia estadística respecto al índice de confiabilidad (sig.)

\section{Regla de decisión para la prueba estadística:}

Si el valor de Tc se ubica fuera de la región aceptada, entonces, se toma como cierta la hipótesis alterna; de lo contrario, se rechaza y se toma como válida la hipótesis nula:

Si p $>0,05 \Rightarrow$ se acepta la Ho

Si $\mathrm{p}<0,05 \Rightarrow$ se rechaza la Ho

\section{Donde:}

$\mathbf{R} \cdot \mathbf{R}=$ Región de Rechazo $\quad$ R.A = Región de aceptación

$\boldsymbol{\alpha} / \mathbf{2}=$ Se denomina valor crítico; es un número que divide la distribución en la región de aceptación y la región de rechazo.

\section{Prueba estadística}

Aplicando la prueba estadística t de Student, se obtuvo el siguiente resultado:

$$
T C=\frac{x_{e}-x_{c}}{\sqrt{\frac{s_{e}^{2}}{n_{e}}}+\frac{s_{c}^{2}}{n_{c}}}=\frac{16.41-12.90}{\sqrt{\frac{0.810}{12}}+\frac{10.921}{11}}=\frac{3.51}{\sqrt{1.059}}=3.41
$$




\section{Regla de decisión}

Como el valor $T C=3.41$ es mayor al valor critico $1.721(0.025)$, lo cual es menor a 0,05 se ha determinado rechazar la hipótesis nula $(\mathrm{Ho})$ y aceptar la hipótesis alterna $(\mathrm{H} \alpha)$. Por tanto, la estrategia “el zorro y las ovejas" si resulta eficaz para la resolución de problemas aditivos.

Resultados de resolución de problemas aditivos, por dimensiones del grupo experimental antes $y$ después del experimento de los niños de la IEP "Juan Bustamante Dueñas" Pusi-2019

La tabla 7, expresa la comparación de los resultados cualitativos obtenidos según la escala de valoración propuesta para el objetivo específico 1:

En la sesión 1, de 12 estudiantes que es el 100\%: se puede apreciar que el $58.33 \%$ se encuentra en la escala valorativa de "siempre", 41.67\% se encuentran en la escala valorativa "a veces" y el 0\% se encuentra en la escala valorativa "nunca"; en la sesión 5, se muestra una mejora positiva ya que el $91.67 \%$ se encuentra en la escala valorativa "siempre", un $8.33 \%$ se encuentra en la escala valorativa "a veces" y un $0 \%$ se encuentra en la escala valorativa "nunca". Finalmente, en la sesión 8, apreciamos que el 100\% de los estudiantes se encuentran en la escala valorativa siempre llegando a la conclusión de que los niños han logrado comprender los problemas que se les ha presentado durante la ejecución de las sesiones de aprendizaje y también en la pos-test.

Tabla 7: Resolución de problemas aditivos con el proceso de comprensión del problema de los estudiantes del grupo experimental.

\begin{tabular}{|c|c|c|c|c|c|c|c|c|c|}
\hline \multirow{3}{*}{ 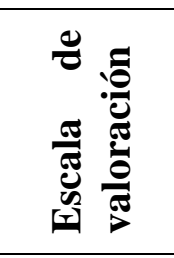 } & \multicolumn{9}{|c|}{ SESIONES } \\
\hline & \multicolumn{3}{|c|}{ Sesión 1} & \multicolumn{3}{|c|}{ Sesión 5} & \multicolumn{3}{|c|}{ Sesión 8} \\
\hline & Fr. & $\%$ & $\begin{array}{l}\% \\
\text { acumul } \\
\text { ado }\end{array}$ & Fr. & $\%$ & $\begin{array}{l}\% \\
\text { acumul } \\
\text { ado }\end{array}$ & Fr. & $\%$ & $\begin{array}{l}\% \\
\text { acum } \\
\text { ulado }\end{array}$ \\
\hline Siempre & 7 & $58.33 \%$ & $58.33 \%$ & 11 & $91.67 \%$ & $91.67 \%$ & 12 & $100 \%$ & $100 \%$ \\
\hline A veces & 5 & $41.67 \%$ & $100 \%$ & 1 & $8.33 \%$ & $100 \%$ & 0 & $0 \%$ & \\
\hline Nunca & 0 & $0 \%$ & & 0 & $0 \%$ & & 0 & $0 \%$ & \\
\hline & 12 & $100 \%$ & & 12 & $100 \%$ & & 12 & $100 \%$ & \\
\hline
\end{tabular}


Tabla 8: Resolución de problemas aditivos con el proceso de búsqueda de estrategias de los estudiantes del grupo experimental.

\begin{tabular}{|c|c|c|c|c|c|c|c|c|c|}
\hline \multirow{3}{*}{ 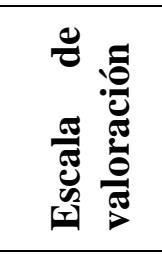 } & \multicolumn{9}{|c|}{ SESIONES } \\
\hline & \multicolumn{3}{|c|}{ Sesión 1} & \multicolumn{3}{|c|}{ Sesión 5} & \multicolumn{3}{|c|}{ Sesión 8} \\
\hline & Fr. & $\%$ & $\begin{array}{c}\% \\
\text { acumu } \\
\text { lado }\end{array}$ & Fr. & $\%$ & $\begin{array}{c}\% \\
\text { acumul } \\
\text { ado }\end{array}$ & Fr. & $\%$ & $\begin{array}{c}\% \\
\text { acum } \\
\text { ulado }\end{array}$ \\
\hline Siempre & 2 & $16.67 \%$ & $\begin{array}{c}16.67 \\
\%\end{array}$ & 10 & $83.33 \%$ & $83.33 \%$ & 12 & $100 \%$ & $100 \%$ \\
\hline A veces & 10 & $83.33 \%$ & $100 \%$ & 2 & $16.67 \%$ & $100 \%$ & 0 & $0 \%$ & \\
\hline Nunca & 0 & $0 \%$ & & 0 & $0 \%$ & & 0 & $0 \%$ & \\
\hline Total & 12 & $100 \%$ & & 12 & $100 \%$ & & 12 & $100 \%$ & \\
\hline
\end{tabular}

En la tabla 8 se muestran los resultados obtenidos en la comparación de los resultados cualitativos obtenidos según la escala de valoración propuesta para el objetivo específico 2:

En la sesión 1, de 12 estudiantes que es el 100\%: podemos observar que el $16.67 \%$ se encuentra en la escala valorativa de "siempre", $83.33 \%$ se encuentran en la escala valorativa "a veces" y el 0\% se encuentra en la escala valorativa "nunca"; en la sesión $\mathrm{N}^{\circ} 5$, también se muestra una mejora positiva ya que el $83.33 \%$ se encuentra en la escala valorativa "siempre", un $16.67 \%$ se encuentra en la escala valorativa "a veces" y un $0 \%$ se encuentra en la escala valorativa "nunca". Finalmente, en la sesión $\mathrm{N}^{\circ} 8$, apreciamos que el $100 \%$ de los estudiantes se encuentran en la escala valorativa siempre llegando a la conclusión de que los niños han logrado mejorar en el proceso de búsqueda de estrategias durante la ejecución de las sesiones de aprendizaje y también en la pos-test. Como platea el ministerio de educación, en las rutas de aprendizaje que para el proceso de búsqueda de estrategias también debemos tomar en cuenta las siguientes preguntas: ¿Este problema es parecido a otros que ya conoces? ¿Podrías plantear el problema de otra forma? Imagínate un problema parecido, pero más sencillo.

En la tabla 9 se refleja los resultados obtenidos en la comparación de los resultados cualitativos obtenidos según la escala de valoración propuesta para el objetivo específico 3:

En la sesión 1, del 100\% de los niños: podemos observar que el $8.33 \%$ se encuentra en la escala valorativa de "siempre", 91.67\% se encuentran en la escala valorativa "a veces" y el $0 \%$ se encuentra en la escala valorativa "nunca"; en la sesión 5 , se muestra que el $83.33 \%$ se encuentra en la escala valorativa "siempre", un $16.67 \%$ se encuentra en la escala valorativa "a veces" y un $0 \%$ se encuentra en la escala valorativa "nunca". 
Finalmente, en la sesión 8, apreciamos que el $100 \%$ de los estudiantes se encuentran en la escala valorativa siempre con estos resultados comprobamos de que los niños han logrado mejorar en el proceso de ejecución de estrategias durante la ejecución de las sesiones de aprendizaje y también en la pos-test.

Tabla 9: Resolución de problemas aditivos con el proceso de ejecución del problema de los estudiantes del grupo experimental.

\begin{tabular}{|c|c|c|c|c|c|c|c|c|c|}
\hline \multirow[b]{3}{*}{$\begin{array}{l}\text { Escala de } \\
\text { valoración }\end{array}$} & \multicolumn{9}{|c|}{ SESIONES } \\
\hline & \multicolumn{3}{|c|}{ Sesión 1} & \multicolumn{3}{|c|}{ Sesión 5} & \multicolumn{3}{|c|}{ Sesión 8} \\
\hline & Fr. & $\%$ & $\begin{array}{l}\% \\
\text { acumul } \\
\text { ado }\end{array}$ & Fr. & $\%$ & $\begin{array}{l}\% \\
\text { acumul } \\
\text { ado }\end{array}$ & Fr. & $\%$ & $\begin{array}{l}\% \\
\text { acumula } \\
\text { do }\end{array}$ \\
\hline Siempre & 1 & $8.33 \%$ & $8.33 \%$ & 10 & $\begin{array}{l}83.33 \\
\%\end{array}$ & $83.33 \%$ & 12 & $\begin{array}{l}100 \\
\%\end{array}$ & $100 \%$ \\
\hline A veces & 11 & $\begin{array}{l}91.67 \\
\%\end{array}$ & $100 \%$ & 2 & $\begin{array}{l}16.67 \\
\%\end{array}$ & $100 \%$ & 0 & $0 \%$ & \\
\hline Nunca & 0 & $0 \%$ & & 0 & $0 \%$ & & 0 & $0 \%$ & \\
\hline Total & 12 & $100 \%$ & & 12 & $100 \%$ & & 12 & $\begin{array}{l}100 \\
\%\end{array}$ & \\
\hline
\end{tabular}

La tabla 10 muestra los resultados obtenidos en la comparación de los resultados cualitativos obtenidos según la escala de valoración propuesta para el objetivo específico 4:

Tabla 10: Resolución de problemas aditivos con el proceso de reflexión y comunicación de los estudiantes del grupo experimental.

\begin{tabular}{|c|c|c|c|c|c|c|c|c|c|}
\hline \multirow{3}{*}{$\begin{array}{l}\text { Escala de } \\
\text { valoración }\end{array}$} & \multicolumn{9}{|c|}{ SESIONES } \\
\hline & \multicolumn{3}{|c|}{ Sesión 1} & \multicolumn{3}{|c|}{ Sesión 5} & \multicolumn{3}{|c|}{ Sesión 8} \\
\hline & Fr. & $\%$ & $\begin{array}{l}\% \\
\text { acumul } \\
\text { ado }\end{array}$ & Fr. & $\%$ & $\begin{array}{l}\% \\
\text { acumu } \\
\text { lado }\end{array}$ & Fr. & $\%$ & $\begin{array}{l}\% \\
\text { acumula } \\
\text { do }\end{array}$ \\
\hline Siempre & 0 & $0 \%$ & $0 \%$ & 8 & $\begin{array}{l}66.67 \\
\%\end{array}$ & $\begin{array}{l}66.67 \\
\%\end{array}$ & 11 & $\begin{array}{l}91.67 \\
\%\end{array}$ & $91.67 \%$ \\
\hline A veces & 12 & $\begin{array}{l}100 \\
\%\end{array}$ & $100 \%$ & 4 & $\begin{array}{l}33.33 \\
\%\end{array}$ & $100 \%$ & 1 & $8.33 \%$ & $100 \%$ \\
\hline Nunca & 0 & $0 \%$ & & 0 & $0 \%$ & & 0 & $0 \%$ & \\
\hline Total & 12 & $\begin{array}{l}100 \\
\%\end{array}$ & & 12 & $100 \%$ & & 12 & $100 \%$ & \\
\hline
\end{tabular}

En la sesión 1, del 100\% de los niños: podemos observar que el $0 \%$ se encuentra en la escala valorativa de "siempre", $100 \%$ de los niños se encuentran en la escala valorativa "a veces" y el 0\% se encuentra en la escala valorativa "nunca"; en la sesión 5, se muestra que el $66.67 \%$ se encuentra en la escala valorativa "siempre", el 33.33\% se encuentra en 
la escala valorativa "a veces" y un $0 \%$ se encuentra en la escala valorativa "nunca". Finalmente, en la sesión 8, apreciamos que el 91.67\% de los estudiantes se encuentran en la escala valorativa "siempre" y $8.33 \%$ se encuentra en la escala valorativa "a veces" con estos resultados comprobamos que el mayor porcentaje de los niños han logrado mejorar en el proceso de reflexión y comunicación durante la ejecución de las sesiones de aprendizaje y también en el pos-test.

Teniendo en cuenta el aporte del ministerio de educación sobre el juego el zorro y las ovejas como estrategia. Se puede utilizar para que el estudiante desarrolle su capacidad de razonamiento pues tiene que pensar en las consecuencias de cada una de sus jugadas e imaginar las posibilidades jugadas de su contrincante cada vez que efectúa un movimiento determinado de alguna de las piezas las que le corresponde jugar. En este sentido, los jugadores usan constantemente las proposiciones implicativas del tipo: "si...entonces"; es decir, utilizan cadenas de razonamiento. Se observa que, de 23 niños que conformaban la muestra de estudio de la Institución educativa primaria $\mathrm{N}^{\circ} 72308$ “juan Bustamante dueñas" los 12 estudiantes del grupo experimental que recibieron la enseñanza de resolución de problemas aditivos con la estrategia "el zorro y las ovejas", demostraron la eficacia de la estrategia ya mencionada, conforme lo demuestran los resultados obtenidos expresados en las tablas, figuras, análisis e interpretación. La prueba de hipótesis comprueba estadísticamente la validez de los resultados con el nivel de significancia alcanzado de ,025 es la razón por el que se aceptó la hipótesis alterna, ya que ha sido menor de 0,05 , por tanto, es confiable la estrategia propuesta, para su aplicación en la mejora de resolución de problemas aditivos de la población de estudio y demás instituciones de la zona.

Se logró determinar, que la estrategia propuesta, es eficaz para la resolución de problemas aditivos de los estudiantes (conforme con las conclusiones realizadas para esta investigación), para lo cual, compartimos los resultados obtenidos por Morales (2011), lo cual afirma: que la estrategia "el zorro y las ovejas" es un valioso aporte al proceso de enseñanza y aprendizaje más adecuado y objetivo y además que Se ha recuperado los juegos andinos, específicamente del "zorro y la oveja" para el aprendizaje de la adición y sustracción en los niños del III Ciclo.

También se comprueba con los resultados que propone Sanizo (2013), mencionado que en el primer grado de educación primaria, el aprendizaje de la matemática debe ser desarrollado o adquirido a través de actividades significativas, con la utilización de estrategias didácticas como el juego, uso de material concreto y otros a fin de favorecer 
el desarrollo de habilidades en los niños para la solución de problemas reales a que se enfrenta y como también desarrollar su capacidad de razonamiento a partir de sus saberes previos también que en la enseñanza de la matemática el docente debe propiciar estrategias innovadoras de acuerdo a la realidad del contexto y las demandas y necesidades de los estudiantes, que motiven la iniciativa, la creatividad, la inventiva de los mismos, a fin de desarrollar habilidades para resolver operaciones y problemas de su contexto o realidad.

\section{CONCLUSIONES}

La estrategia "el zorro y as ovejas" es eficaz en la resolución de problemas aditivos en niños de segundo grado de la IEP “Juan Bustamante Dueñas” Pusi-2019 que lograron elevar el nivel de aprendizaje ya que el grupo control obtuvo en la prueba de salida un promedio total de $10.29 \mathrm{y}$ el grupo experimental al que se le aplico el experimento logro elevar sus promedios a 16.41, esto se vio en la prueba de salida comprobado con la prueba T de Student. Con un valor critico de $1.721(0,025)$ que es menor a 0,05, aceptando así la hipótesis alterna.

La estrategia "el zorro y as ovejas" es eficaz para a comprensión de problemas aditivos, los niños con ayuda del material y las fichas del juego han logrado entender mejor los problemas eso lo demostraron en el transcurso de las sesiones.

La estrategia "el zorro y as ovejas" resulta eficaz como estrategia escogida por los estudiantes para la resolución de problemas que se les ha presentado en cada sesión de aprendizaje.

La estrategia "el zorro y as ovejas" les resulta eficaz a los estudiantes al momento de ejecutar la estrategia para resolver un problema aditivo ya que los problemas que han desarrollado estuvieron adecuados para desarrollar con más facilidad con el material.

La estrategia “el zorro y as ovejas" les resulta eficaz para el proceso didáctico de reflexión y comunicación ya que después de desarrollar los problemas aditivos, entre los estudiantes reflexionan sobre la importancia de este juego.

\section{AGRADECIMIENTOS}

Un especial agradecimiento a la institución educativa primaria "Juan Bustamante Dueñas" de Pusi, en especial a los estudiantes que participaron en la presente investigación.

\section{REFERENCIAS} Personal Social en los niños (as) de cuatro años de la IEI Corazon de Jesus Acora 2017. Puno: UNAP. 


\section{LA ESTRATEGIA “EL ZORRO Y LAS OVEJAS” EN LA RESOLUCIÓN DE PROBLEMAS ADITIVOS EN NIÑOS Y \\ NIÑAS DEL SEGUNDO GRADO DE PRIMARIA \\ ISSN: 2789-0309 ISSN-L: 2789-0309}

Carbajal, M. R., \& Pozo, G. C. (2019). La etnomatemática y el desarollo del pensamiento logico matemático en los alumnos $5^{\circ}$ grado de educación primaria en la I.E. 34116 de Yanacocha Yanahuanca-Pasco 2017. Yanahuanca: Universidad Nacional Daniel Alcides Carrión.

Charaja, F. (2018). El MAPIC en la Investigación Científica. Puno: Corporación SIRIO EIRL.

Condor, T. M. (2019). Los juegos tradicioales como estrategia en e aprendizaje de la matematica en os estudiantes del segundo grado de primaria de la Institucion Educativa N²0326 Puqio Cano Hualmay,2016. Huacho: Universidad Nacional José Faustino Sánchez Carrión.

De Educación, M. (2015). Matemáticas En Educación Intercultural Bilingüe. 145.

De Guzmán, M. (2007). Enseñanza De Las Ciencias Y La Matemática. Revista Iberoamericana De Educación N. ${ }^{\circ} 43,58$.

DRE. (2018). Evaluación Censal de Estudiantes DRE-Puno. ¿Que aprendizajes lograron nuestros estudiantes?

Gabarrete, M. E. (2013). La etnomatemática como campo de investigación y acción didactica: su evlución y recursos para la formacion de profesores desde la equidad. Costa Rica: Revista Latinoamerica de Etnomatematica.

Gastelu, L. S., \& Padilla, D. P. (2017). Influencia de juegos didacticos en el aprendizaje del area de matematica en los alumnos de la Institucion Educativa, Huaycan. Lima: Uiversidad Nacional de Educacion.

Gentile Lafaille, M. E. (2007). Notas sobre algunas mujeres del collasuyu (siglos XV al XVIII). Buenos Aires: CONICET.

Godino , J. D., Batanero, C., \& Font, V. (2003). fundamentos de la enseñanza y el aprendizaje de las matematicas para maestros. España: Edumat-Maestros.

Hernández, R., Fernández, C., \& Baptista, M. d. (2014). Metodología de la Investigación. México D. F.: McGRAW-HILL / INTERAMERICANA EDITORES, S.A. DE C.V.

Holm, O. (1945). Taptana o el ajedrez de Atahualpa: a loa años 425 de Cajamarca. Cuadernos de historia y arqueología. Guayaquil : casa de la cultura ecuatoriana .

Llalla, S., \& Yanque, C. (2015). Juegos andinos como medio para optimizar las relaciones interpersonales en niños y niñas de la IE 56039 de Tinta y 56038 de Cuchuma de los años 2009 al 2012. Arequipa: Universidad Nacional De San Agustin .

MINEDU. (2008). Historias para ver y vivir el presente . Perú: Biblioteca Nacional del Perú.

MINEDU. (2015). Matemáticas en educación intercultural bilingüe. Lima-Peru: Franco EIRL.

MINEDU. (2015). Rutas del aprendizaje . Lima: Ministerio de Educación.

MINEDU. (2017). Programa curricular de Educacion Primaria. Lima: Ministerio de Educación.

MINEDU. (2018). Evaluación Censal de Estudiantes. ¿Que aprendizajes lograron nuestros estuduantes?, 24.

Morales, P. M. (2011). Importancia del juego "el zorro y la oveja" para el aprendizaje dela adicion y sustraccion en los niños y niñas del III ciclo de la institucion educativa $N^{\circ} 72160$ de Collpani. Puno: UNAP.

NAVARRO BURGOS, E. (2015). Aplicación De Estrategias Lúdicas Para El Mejoramiento Del Aprendizaje De La Matemática De Los Estudiantes Del Primer Grado De Secundaria De La I.E “Absalón Vásquez Villanueva” Del Caserío La Shita - JESÚS - 2014. Cajamarca - Perú.

OCDE. (2018). Base de datos PISA 2018. París: OCDE. 
Otzen, T., \& Manterola, C. (2017). Técnicas de Muestreo sobre una Población a Estudio. Int. J. Morphol., $35(1), 227-232$.

Regular, D. G. (2019). Currículo Nacional. 116.

Sanizo, G. M. (2013). "El juego y manipulacion de material concreto como estrategias didacticas para la resolucion de ejercicios y problemas en el area de matematica intercultural en el primer grado de la IEP N 70035 Bellavista - Puno 2012". Puno: UNAP.

UNICEF. (2018). Aprendizaje a través del juego . New York: UNICEF.

Venero, A. (2012). Matemática Básica (2ºd.). Lima: Ediciones Gemar.

Werlich, E. (1982). A. Text Grammar of English. Heidelberg: Quelle and Meyer. 